\title{
Calidad de vida de personas con discapacidad intelectual en proceso de envejecimiento en entornos de vivienda
}

\section{Quality of life of individuals with intellectual disability who are aging in supported living environments}

\begin{abstract}
Resumen
El trabajo que se presenta responde a la necesidad urgente de identificar y comprender posibles necesidades de las personas con discapacidad intelectual que envejecen para mejorar la prestación de apoyos a este colectivo. Para ello, se analiza la calidad de vida de los usuarios del servicio de viviendas de Atzegi $(N=174)$, entre los cuales un $48 \%$ tiene más de 50 años. Se utilizó la Escala Integral de Calidad de Vida (Verdugo et al., 2009) al ser el instrumento de evaluación que se emplea de manera sistemática en el servicio. Los resultados ponen de manifiesto que no existen diferencias estadísticamente significativas entre personas en función de su edad, pero al tener en cuenta otras variables sociodemográficas la actividad laboral aparece como aspecto especialmente relevante en la calidad de vida percibida por los mayores, motivo por el que se discuten futuras líneas de acción a adoptar por las organizaciones.
\end{abstract}

\section{Palabras clave}

Calidad de vida, discapacidad intelectual, envejecimiento, vivienda con apoyos.

\section{Nerea Otamendi Ormazabal $<$ nerea@atzegi.org>}

Atzegi. Asociación Guipuzcoana en favor de las personas con discapacidad intelectual. España

Patricia Navas Macho <patricianavas@usal.es> Universidad de Salamanca. España

\begin{abstract}
This research study addresses the need for a comprehensive identification of possible needs experienced by individuals with intellectual disabilities who are aging to improve service provision. For this purpose, the quality of life of Atzegi's supported accommodation service users is analyzed $(\mathrm{N}=174)$, among which $48 \%$ are over 50 years old. The Integral Quality of Life Scale (Verdugo et al. 2009) was applied as it is the assessment instrument systematically used in the service. The results show that there are no statistically significant differences between people according to their age. However, when taking into account other sociodemographic variables, work activity appears as an especially relevant aspect in the quality of life perceived by the elderly, reason why future lines of action are discussed.
\end{abstract}

\section{Keywords}

Quality of life, intellectual disability, aging, supported accommodation.

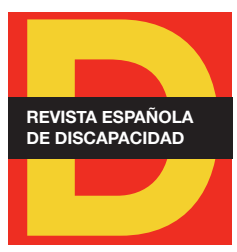

Para citar:

Otamendi, N. y Navas, P. (2018):

"Calidad de vida de personas con discapacidad intelectual en proceso de envejecimiento en entornos de vivienda". Revista Española de Discapacidad, 6 (II): 27-47.

Doi: <https://doi.org/10.5569/23405104.06.02.02>

Fecha de recepción: 23-01-2018 Fecha de aceptación: 27-11-2018 


\section{Introducción ${ }^{1}$}

Las profundas transformaciones que han surgido durante los últimos años en la prestación de apoyos a las personas con discapacidad intelectual (DI) han dado lugar a un aumento considerable de su esperanza de vida. Las estadísticas de países como España (Baroja et al., 2014) señalan que, dentro de una década, más del $60 \%$ de las personas con DI tendrán más de 45 años. En Europa, el 45,4 \% del total de población con discapacidad está en proceso de envejecimiento (Grammenos, 2013). En Estados Unidos se ha documentado un incremento en la esperanza de vida en algunos colectivos (i.e., síndrome de Down) del $400 \%$ desde 1960 (Presson et al., 2013). Estos datos explican por qué el Comité de Rehabilitación e Integración de las Personas con Discapacidad del Consejo de Europa llegó a definir el fenómeno del envejecimiento en población con DI como una "emergencia silenciosa" (Aguado y Alcedo, 2004: 261), una nueva realidad social que ha cogido desprevenidos a los responsables de los servicios sociales y sanitarios (Berjano y García, 2010), representando un gran desafío demográfico para la población mundial (Reppermund y Trollor, 2016).

Las organizaciones de nuestro país se están encontrando con ciertas dificultades para dar respuesta a las necesidades que están surgiendo en el colectivo de personas con DI que envejece, al tener un vago conocimiento sobre el mismo (Grupo de Trabajo de Envejecimiento Activo del CERMI Estatal, 2012). Estas dificultades no son exclusivas del contexto español, sino que se extienden a otros países. Entre las mismas podemos destacar, las siguientes: comienzan a surgir en las personas con DI por primera vez, y de manera más temprana (Haveman et al., 2010; Heller y Sorensen, 2013), problemas de salud asociados al proceso de envejecimiento (Navas et al., 2014; Reppermund y Trollor, 2016; Wark et al., 2016) entre los que preocupa especialmente el deterioro cognitivo (Jokinen et al., 2013). Surge asimismo la necesidad de planificar tanto la transición hacia la jubilación por el impacto que el cese de la actividad laboral pudiera tener en la calidad de vida de las personas (Elorriaga et al., 2012; Stancliffe et al., 2013) como el posible cambio a otros entornos de vivienda ante la pérdida de los cuidadores principales (Bigby, 2008), ofreciendo alternativas habitacionales acordes a los deseos de la persona (Novell et al., 2008). Proporcionar apoyos durante el proceso de duelo (Brickell y Munir, 2008; Cristóbal et al., 2017) y seguir fomentando su inclusión social a pesar de la posible pérdida de figuras de apoyo como los familiares (Berzosa, 2013), son líneas de actuación generalmente señaladas en la literatura para seguir fomentando el bienestar emocional de las personas con DI a medida que envejecen. La importancia de respetar los principios que inspiran la Planificación Centrada en la Persona (PCP) durante la última etapa del ciclo vital, también está acaparando buena parte de la atención de investigadores (e.g., Heller et al., 2011; Kingsbury, 2009; Kirkendall et al., 2017; Wiese et al., 2014) y organizaciones, como así pone de manifiesto la iniciativa AGID (Developing Training Modules for staff on Ageing and Disability Issues) financiada por la Comisión Europea, o las acciones llevadas a cabo por organizaciones de nuestro país en materia de Planificación Personal por Adelantado, PPA (Carratalá et al., 2017).

Dar una respuesta adecuada a estas y otras necesidades desde el ámbito organizacional permitirá seguir trabajando en la mejora de calidad de vida de las personas con DI.

\footnotetext{
1. Este artículo se ha desarrollado en el marco del proyecto de investigación I+D PSI2015-64517-R financiado por el Ministerio de Economía y Competitividad dentro de las ayudas para la realización de proyectos de investigación dentro del Programa Estatal de Investigación, Desarrollo e Innovación Orientada a los Retos de la Sociedad, Convocatoria 2015. Los autores quisieran agradecer a los profesionales de Atzegi, las personas con DI y sus familiares su participación en el mismo.
} 
Pese a la complejidad que entraña definir un concepto de carácter subjetivo como es el de calidad de vida (CdV), algunas definiciones, como la propuesta por Schalock y Verdugo (2002), facilitan su comprensión. Según estos autores, entendemos la CdV como un estado deseado de bienestar personal que es multidimensional, tiene propiedades universales y ligadas a la cultura, tiene componentes objetivos y subjetivos, y se ve influido por factores personales y ambientales.

El significado del constructo CdV se ha debatido durante muchos años, con más de 200 definiciones encontradas en la revisión inicial de este concepto (Schalock y Verdugo, 2002). Tras su introducción en el campo de las discapacidades intelectuales y del desarrollo, el interés por este término se centró no tanto en definir qué entendemos por $\mathrm{CdV}$, sino en establecer sus dimensiones básicas e indicadores asociados, con objeto de poder hacer realidad su evaluación y medida en las organizaciones, bajo la premisa de que la CdV es más un fenómeno multidimensional que un estado vital singular. De este modo, Schalock y Verdugo (2002) apuestan por un modelo multidimensional de CdV constituido por ocho dimensiones cuya evaluación ha de tener en cuenta lo que la persona con DI valora como relevante e importante en su vida (Schalock et al., 2006): bienestar físico, emocional y material, relaciones interpersonales, inclusión social, desarrollo personal, derechos y autodeterminación.

La implementación de paradigmas orientados a la mejora de CdV dentro de las organizaciones que prestan apoyo a personas con DI, ha sido determinante para que la esperanza de vida de este colectivo haya aumentado significativamente en los últimos años. Los procesos de desinstitucionalización, que respondieron a la necesidad de una mayor presencia en la comunidad de las personas con DI, y el paso a un paradigma de apoyos centrado en mejorar el funcionamiento de las mismas en todos los ámbitos de su vida (Schalock y Verdugo, 2010), han posibilitado grandes avances en la prestación de servicios socio-sanitarios y han permitido que, a día de hoy, nos enfrentemos a un futuro no muy lejano en el que contemos con una gran parte de la población con DI envejecida. En este sentido, cabe destacar que, aunque no se pueda determinar con claridad la edad en la que comienza el proceso de envejecimiento en las personas con DI, la literatura científica en el campo de las discapacidades intelectuales y del desarrollo tiende a señalar que su inicio se encuentra entre los 45 y 50 años, debiendo tener en cuenta que el envejecimiento es un proceso continuo y dinámico que se dibuja a lo largo de la vida y que vendría dado, no tanto por el alcance de una edad cronológica concreta, sino por el declive funcional observado (Madrigal et al., 2007).

Este declive funcional pudiera afectar al modo en que las organizaciones prestan apoyo en distintas áreas de la vida de la persona, y de manera especial en el contexto de vivienda. Esta situación comienza a observarse en la Asociación Guipuzcoana en favor de las personas con discapacidad intelectual (Atzegi), entorno en el que se lleva a cabo el presente estudio. Atzegi cuenta ya con un gran número de personas mayores en su servicio de viviendas, viviendas integradas en la comunidad que difieren en cuanto al grado de apoyo que se presta en las mismas. Para ser exactos, de las 217 personas usuarias de este servicio, 111 (un $51 \%$ ) tienen más de 50 años. Además, es importante destacar que, en los últimos años, la media de las nuevas incorporaciones se encuentra en los 43 años de edad. Este hecho ha dado lugar a numerosos debates sobre la manera de atender a este colectivo, y a pesar de que Atzegi desde hace tiempo viene trabajando bajo el paradigma de CdV a través de la PCP, resulta preciso identificar qué variables pudieran influir en la CdV de aquellas personas con DI que envejecen en el servicio de viviendas, con el objetivo último de reflexionar sobre la idoneidad o no de ofrecer apoyos en el entorno en que las personas se encuentran en la actualidad u optar por otro tipo de recursos (e.g., residencias específicas). 
En este sentido, la Confederación Española de Organizaciones en favor de las Personas con Discapacidad Intelectual, FEAPS (ahora Plena Inclusión) defiende que 'el modelo más eficaz es el que adecua los apoyos y servicios a la realidad de las personas, en el mismo lugar donde están y sin crear servicios segregados especializados alejados del contexto donde el sujeto ha crecido y ha vivido durante muchos años' (Pereyra, 2007: 22).

La investigación también subraya que las personas con DI en proceso de envejecimiento experimentan mayor satisfacción y resultados personales más valorados cuando pueden seguir residiendo en la comunidad y reciben ayuda por parte de servicios específicos cuando la situación así lo requiere (Higgins y Mansell, 2009), sin necesidad de crear nuevos recursos de carácter más asistencial. No obstante, y si bien los resultados positivos de la vida en comunidad son incuestionables (véase al respecto Bigby et al., 2012; Mansell, 2006; Mansell, 2010; entre otros) estudios recientes ponen de manifiesto que el tipo de residencia (e.g., centro residencial vs. vivienda con apoyos en la comunidad) puede no ser una variable tan determinante como el tipo de apoyo que se presta a la hora de conseguir un incremento en el bienestar emocional de la persona (Beadle-Brown et al., 2015; Bigby y Beadle-Brown, 2018), un apoyo que ha de ser activo, centrado en la persona y sus potencialidades y que favorezca la participación en actividades de interés para la persona con DI.

Las características estructurales del modelo de viviendas de Atzegi, pudieran generar dificultades a la hora de ofrecer este tipo de atención a aquellos que, a consecuencia del proceso de envejecimiento, pudieran requerir apoyos más intensos. Así, Atzegi actualmente cuenta con 31 viviendas repartidas por todo el territorio de Guipúzcoa. Estas viviendas se clasifican en tres tipos según la intensidad de los apoyos que precisan quienes las habitan: vivienda de atención intensa (integradas por un máximo de ocho usuarios que cuentan con apoyo de tres profesionales de lunes a viernes en horario de tarde y 24 horas durante los fines de semana); vivienda de atención media (integradas por un máximo de ocho usuarios que cuentan con apoyo de dos profesionales de lunes a viernes en horario de tarde y 24 horas durante los fines de semana); y vivienda de atención ligera o intermitente (integradas por un máximo de cinco usuarios que cuentan con apoyo de dos profesionales de lunes a viernes en horario de tarde, uno de los cuales solo acude dos o tres tardes a la semana). Todas las viviendas ofrecen atención directa desde la tarde hasta la mañana siguiente, lo que obliga a las personas con Dl que residen en las mismas a desarrollar una actividad diurna. El simple hecho de mantener el ritmo que exige esta estructura puede ser un obstáculo para las personas con DI en proceso de envejecimiento, ocasionando muchas veces el traslado de las mismas a residencias de tercera edad. Por este motivo resulta imprescindible conocer cómo podemos apoyar a las personas con DI a medida que envejecen, identificando aquellas variables que pudieran ejercer un impacto negativo en la CdV de las personas en esta última etapa de la vida, con el objetivo último de ofrecer apoyos apropiados en su entorno habitual que minimicen un posible descenso de su bienestar.

\section{Metodología}

Se realizó un estudio de carácter cuantitativo para analizar el impacto que los cambios asociados a la vejez pudieran generar en cada una de las dimensiones que integran una vida de calidad, comparando resultados de población joven $(\mathrm{N}=91)$ con población mayor $(\mathrm{N}=83)$ que, en el momento de la investigación, residía en 
el servicio de viviendas de Atzegi, persiguiendo los siguientes objetivos específicos: (1) detectar diferencias en CdV medida de manera objetiva y subjetiva entre personas con DI mayores y menores de 50 años usuarias del servicio de viviendas de Atzegi; (2) explorar diferencias en CdV medida de manera objetiva y subjetiva entre las personas con DI mayores y menores de 50 años en función de una serie de variables (entorno de vivienda, sexo, actividad ocupacional, etc.) que pudieran afectar a la consecución de resultados personales valorados; y (3) detectar, a partir de los resultados anteriores, qué dimensiones de CdV son susceptibles de mejora en las personas con DI mayores de 50 años a quienes se presta apoyos desde la entidad.

\section{Participantes}

Si bien el servicio de viviendas de Atzegi cuenta con 217 usuarios, el presente trabajo evaluó la calidad de vida de 197 personas, dado que las 20 restantes se habían incorporado recientemente al servicio, no disponiendo los profesionales de suficiente información sobre las mismas como para realizar una valoración objetiva de su CdV. Con ocho de estas 197 personas no se pudo aplicar la parte subjetiva de la Escala Integral de Calidad de Vida (Verdugo et al., 2009) -instrumento utilizado en este estudio y en el que la propia persona con DI puede ofrecer su percepción sobre el estado actual de su CdV si cuenta con ciertas habilidades de comprensión y expresión-, bien por presentar especiales dificultades de comunicación que impidieron su cumplimentación incluso mediante el empleo de ayudas visuales o pictogramas, o bien por negarse a participar en la evaluación, respetando en este último caso su decisión.

Además, el análisis de los residuos estandarizados puso de manifiesto que 23 casos (11,6\%) mostraron valores absolutos significativamente superiores a 3,29 y 2,58 en varias dimensiones de CdV. Con el objetivo de que estos outliers no distorsionaran los datos, fueron eliminados de la muestra que, finalmente, contó con 174 casos cuyas características se exponen en la Tabla 1.

A pesar de haber establecido cuatro categorías de edad durante el proceso de recogida de datos (de 18 a 30 años, de 31 a 49 años, de 50 a 65 años y más de 65 años) y teniendo presente que solo ocho personas contaban con más de 65 años, se establecieron dos categorías de edad (menores y mayores de 50 años) para analizar posibles diferencias en lo que a CdV se refiere entre personas con DI en función de su edad, teniendo en cuenta asimismo que la literatura científica señala el inicio del declive funcional de este colectivo en torno a los 45 años de edad.

Los usuarios del servicio de viviendas de Atzegi se caracterizaron, en general, por residir en viviendas en las que se prestan apoyos de carácter intenso o medio, independientemente de su edad, realizar algún tipo de actividad diurna, tener modificada su capacidad legal de obrar y presentar un porcentaje de discapacidad superior al $65 \%$. No obstante, observamos diferencias entre personas mayores y menores de 50 años que merece la pena comentar pues pudieran tener un impacto significativo en su CdV. Así, se puede apreciar que una gran parte de las personas mayores de 50 años $(\mathrm{N}=39)$ acudía a centros de día para personas con $\mathrm{DI}$, mientras que, en las personas menores de 50 años, fueron el taller ocupacional y el empleo las actividades más comunes durante el día.

En lo que a su tutela se refiere, la gran mayoría de usuarios mayores de 50 años estaban tutelados por algún familiar, dato que pudiera reflejar el resultado de una época en la que de manera sistemática se aconsejaba 
a las familias incapacitar legalmente a la persona con DI. Con respecto al grado de discapacidad reconocido, si bien los resultados no llegaron a ser significativos, aquellos de mayor edad presentaron porcentajes de discapacidad mayores.

\begin{tabular}{|c|c|c|c|c|c|c|}
\hline & & $\begin{array}{c}\text { Menores de } 50 \\
\text { años } \\
(\mathrm{N}=91)\end{array}$ & $\begin{array}{l}\text { Mayores de } 50 \\
\text { años } \\
(\mathrm{N}=83)\end{array}$ & Total & $x^{2}$ & $\mathbf{p}$ \\
\hline \multirow{2}{*}{ Sexo } & Hombre & $46(50,5 \%)$ & $42(50,6 \%)$ & 88 & \multirow{2}{*}{, 00} & \multirow{2}{*}{,99 } \\
\hline & Mujer & $45(49,5 \%)$ & $41(49,4 \%)$ & 86 & & \\
\hline \multirow{3}{*}{ Tipo de vivienda } & Intermitente & $18(19,8 \%)$ & $13(15,6 \%)$ & 31 & \multirow{3}{*}{4,19} & \multirow{3}{*}{,123 } \\
\hline & Media & $51(56,0 \%)$ & $38(45,8 \%)$ & 89 & & \\
\hline & Intensa & $22(24,2 \%)$ & $32(38,6 \%)$ & 54 & & \\
\hline \multirow{3}{*}{ Actividad diurna } & Empleo & $32(35,2 \%)$ & $24(28,9 \%)$ & 56 & & \\
\hline & Ocupacional & $36(39,6 \%)$ & $20(24,1 \%)$ & 56 & & \\
\hline & Centro de Día & $23(25,3 \%)$ & $39(47,0 \%)$ & 62 & & \\
\hline \multirow{3}{*}{ Tutela } & No tutelado & $21(23,1 \%)$ & $15(18,1 \%)$ & 36 & \multirow{3}{*}{7,14} & \multirow{3}{*}{,02 } \\
\hline & Fundación tutelar & $32(35,2 \%)$ & $17(20,5 \%)$ & 49 & & \\
\hline & Tutela familiar & $38(41,8 \%)$ & $51(61,4 \%)$ & 89 & & \\
\hline \multirow{4}{*}{$\begin{array}{l}\text { Tiempo en el } \\
\text { servicio }\end{array}$} & 1-5 años & $27(29,7 \%)$ & $16(19,3 \%)$ & 43 & \multirow{4}{*}{10,46} & \multirow{4}{*}{,01 } \\
\hline & 6-15 años & $46(50,5 \%)$ & $38(45,8 \%)$ & 84 & & \\
\hline & 16-22 años & $14(15,4 \%)$ & $13(15,7 \%)$ & 27 & & \\
\hline & +22 años & $4(4,4 \%)$ & $16(19,3 \%)$ & 20 & & \\
\hline \multirow{4}{*}{ Entorno } & Menos de 7.000 habitantes & $18(19,8 \%)$ & $19(22,9 \%)$ & 37 & \multirow{4}{*}{,302 } & \multirow{4}{*}{,96 } \\
\hline & Entre 7.000 y 30.000 habitantes & $23(25,3 \%)$ & $20(24,1 \%)$ & 43 & & \\
\hline & Entre 30.000 y 70.000 habitantes & $10(11,0 \%)$ & $8(9,6 \%)$ & 18 & & \\
\hline & +70.000 habitantes & $40(44,0 \%)$ & $36(43,4 \%)$ & 76 & & \\
\hline \multirow{4}{*}{ \% Discapacidad } & Entre $33 \%$ y $50 \%$ & $14(15,4 \%)$ & $10(12,0 \%)$ & 24 & \multirow{4}{*}{7,13} & \multirow{4}{*}{,06 } \\
\hline & Entre $51 \%$ y $64 \%$ & $7(7,7 \%)$ & $10(12,0 \%)$ & 17 & & \\
\hline & Entre 65 y $74 \%$ & $32(35,2 \%)$ & $16(19,3 \%)$ & 48 & & \\
\hline & $75 \%$ o mas & $38(41,8 \%)$ & $47(56,6 \%)$ & 85 & & \\
\hline \multirow{2}{*}{$\begin{array}{l}\text { Otras } \\
\text { discapacidades }\end{array}$} & Sí & $52(42,9 \%)$ & $37(55,4 \%)$ & 89 & \multirow{2}{*}{2,74} & \multirow{2}{*}{, 09} \\
\hline & No & $39(57,1 \%)$ & $46(44,6 \%)$ & 85 & & \\
\hline
\end{tabular}

Fuente: elaboración propia. 


\section{Instrumento}

En el presente trabajo se empleó la metodología cuantitativa, ya que se analizaron los datos obtenidos a partir de la Escala Integral de Calidad de Vida (Verdugo et al., 2009).

Esta escala tiene como finalidad identificar el perfil de CdV de una persona, medido de manera objetiva y subjetiva, para después poder realizar planes individualizados de apoyo. Permite así estudiar la relación o discrepancia entre lo que es considerado como relevante para la persona y la valoración realizada por el profesional o familiar que la conoce. Se creó, en este sentido, como respuesta al debate surgido algunos años atrás acerca de la relación entre los aspectos objetivos y subjetivos de calidad de vida (Verdugo et al., 2009).

Es importante destacar que la Escala Integral de Calidad de Vida se desarrolló sobre la base del modelo de calidad de vida de ocho dimensiones de Schalock y Verdugo (2002). Tanto la escala objetiva como la subjetiva, cuentan con ítems que actúan como indicadores de estas ocho dimensiones. No obstante, no comparten una estructura idéntica, en tanto que la parte subjetiva incluye la dimensión de bienestar familiar, no contemplada en la parte objetiva. El motivo, según los propios autores, es que, en la mayor parte de los casos, los profesionales no disponen de información precisa sobre las relaciones familiares de las personas con DI (Verdugo et al., 2009:14).

En total, la escala objetiva cuenta con 23 ítems enunciados en tercera persona que presentan un formato de respuesta dicotómico (sí/no). La escala subjetiva está integrada por un total de 39 ítems, enunciados en primera persona y en un formato de respuesta tipo Likert de cuatro puntos: "totalmente en desacuerdo", "en desacuerdo", "de acuerdo" y "totalmente de acuerdo".

Cada dimensión presenta una distribución de puntuaciones estándar con $M=10$ y $D T=3$. A partir de las puntuaciones obtenidas en cada dimensión, es posible calcular un índice de CdV, con $M=100$ y $D T=15$. La cumplimentación completa de la Escala Integral oscila entre los 27-40 minutos (7-10 minutos la escala objetiva y 20-30 minutos la escala subjetiva). Sus propiedades psicométricas indican que permite obtener datos fiables y válidos sobre la CdV de las personas con DI desde una perspectiva tanto objetiva como subjetiva (Verdugo et al., 2009).

La Escala Integral de Calidad de Vida es el instrumento utilizado actualmente en el servicio de viviendas de Atzegi, aplicándose cada dos años para la comisión de vivienda bianual, en la que se exponen los planes personales de apoyo de cada usuario. Este es el motivo por el que en el presente trabajo se empleó este instrumento y no otros de características similares desarrollados con posterioridad, como la Escala INICOFEAPS (Verdugo et al., 2013).

\section{Procedimiento}

El presente trabajo se desarrolló en la organización Atzegi, concretamente en el servicio de viviendas, durante los años 2015 a 2017.

En primer lugar, se llevó a cabo una reunión con los distintos responsables de las viviendas para explicarles la finalidad de la investigación, quienes a su vez informaron a los educadores y personas residentes y 
tutores legales, en su caso. Si bien resulta difícil garantizar que todas las personas comprendieron toda la información, se adaptó la explicación sobre el objetivo de esta investigación a las necesidades de cada persona (e.g., lectura fácil), asegurando el anonimato de los datos y enfatizando que el estudio se orientaba a la consecución de mejoras en cómo los apoyos son prestados dentro del servicio. Solo a partir de este momento se procedió a la solicitud y firma del consentimiento informado, obteniéndolo en todos los casos.

La parte objetiva de la escala fue cumplimentada por profesionales de atención directa del servicio que conocían en profundidad a la persona con DI cuya CdV se pretendía valorar y tenían la posibilidad de observarla en distintos contextos de su vida diaria. Las 174 personas con DI que conforman la muestra de este estudio, cumplimentaron la parte subjetiva, contando para ello con el apoyo de un facilitador. El facilitador fue, en todos los casos su persona de referencia, quien mejor conoce las formas de comunicación y expresión de la persona y quien participa en la elaboración de su plan personal de apoyo. En el caso de personas con ciertas dificultades de comunicación, pero con las habilidades de expresión y comprensión exigidas por el instrumento utilizado, los profesionales hicieron uso de fotografías y pictogramas que facilitaron la comprensión de los enunciados de los ítems.

\section{Análisis}

Los datos del presente trabajo impidieron la realización de contrastes paramétricos ya que todas las variables, salvo la puntuación total de CdV medida de manera subjetiva, presentaron una distribución no normal. Se emplearon por este motivo contrastes no paramétricos que, además, son aconsejables para muestras de pequeño tamaño (Whitley y Ball, 2002): $U$ de Mann-Whitney (para el cálculo de posibles diferencias de medias entre dos muestras independientes); rangos de Wilcoxon (para el cálculo de posibles diferencias de medias entre dos muestras relacionadas); y $H$ de Kruskal-Wallis (para el cálculo de diferencias entre grupos en variables ordinales con más de dos categorías de respuesta). El estadístico chi-cuadrado se utilizó para determinar la posible existencia de relación estadísticamente significativa entre variables categóricas.

Todos los datos fueron analizados mediante el programa estadístico SPSS 21.0.

\section{Resultados}

Se presentan en primer lugar los resultados en CdV medida de manera objetiva y subjetiva en personas con DI mayores y menores de 50 años usuarias del servicio de viviendas de Atzegi, para posteriormente analizar la influencia de una serie de variables (entorno de vivienda, sexo, actividad ocupacional, etc.) en la CdV de los usuarios de los distintos grupos de edad. Los resultados de estos análisis, permitirán con posterioridad detectar si existen dimensiones de CdV susceptibles de mejora en las personas con DI mayores de 50 años a quienes se presta apoyos desde la entidad y qué cambios asociados al proceso de envejecimiento (i.e., jubilación) pudieran estar afectando a esas dimensiones. 
Diferencias en calidad de vida percibida de forma objetiva y subjetiva en los distintos grupos de edad

En primer lugar, se presenta el análisis general de las puntuaciones estándar de CdV obtenidas a partir de la Escala Integral (Verdugo et al., 2009). Tal y como aparece reflejado en la Tabla 2, las personas con DI puntuaron su CdV de forma más baja que los profesionales que les prestaban apoyo, obteniendo además puntuaciones estándar que se encontraban por debajo de la media en todas las dimensiones de CdV y en el índice general de CdV. Esta tendencia se observó tanto en personas mayores de 50 años como en aquellos más jóvenes.

\begin{tabular}{|c|c|c|c|c|c|c|}
\hline & \multicolumn{3}{|c|}{$\begin{array}{l}\text { Menores de } 50 \text { años } \\
(\mathrm{N}=91)\end{array}$} & \multicolumn{3}{|c|}{$\begin{array}{l}\text { Mayores de } 50 \text { años } \\
\quad(\mathrm{N}=83)\end{array}$} \\
\hline & Mínimo & Máximo & Media & Mínimo & Máximo & Media \\
\hline Escala Objetiva AU & 5 & 14 & 10,4 & 5 & 14 & 10,6 \\
\hline Escala Objetiva IS & 2 & 12 & 9,8 & 2 & 12 & 10,2 \\
\hline Escala Objetiva BL & 5 & 12 & 10,7 & 6 & 12 & 10,8 \\
\hline Escala Objetiva BM & 4 & 13 & 8,8 & 4 & 12 & 9,3 \\
\hline Escala Objetiva BEF & 4 & 12 & 10,5 & 4 & 12 & 10,5 \\
\hline Escala Objetiva índice CdV & 67 & 164 & 101,2 & 73 & 118 & 101,5 \\
\hline Escala Subjetiva AU & 4 & 14 & 9,5 & 4 & 15 & 9,3 \\
\hline Escala Subjetiva IS & 2 & 20 & 9,6 & 1 & 15 & 9,6 \\
\hline Escala Subjetiva BL & 1 & 13 & 8,9 & 1 & 13 & 9,1 \\
\hline Escala Subjetiva BM & 1 & 14 & 8,7 & 3 & 14 & 8,5 \\
\hline Escala Subjetiva BEF & 1 & 13 & 8,9 & 2 & 13 & 8,7 \\
\hline Escala Subjetiva BF & 1 & 13 & 8,9 & 0 & 13 & 8,2 \\
\hline Escala Subjetiva índice CdV & 58 & 118 & 94,2 & 42 & 120 & 92,5 \\
\hline
\end{tabular}

AU: autodeterminación; IS: inclusión social; BL: bienestar laboral; BM: bienestar material; BEF: bienestar emocional y físico; BF: bienestar familiar.

Fuente: elaboración propia.

Estas discrepancias entre la valoración personal y profesional resultaron estadísticamente significativas. Así, y frente a la valoración realizada por los profesionales, ambos grupos de edad puntuaron de forma significativamente más baja su CdV general, su autodeterminación, el bienestar laboral y el bienestar emocional y físico (Tabla 3), no habiendo observado diferencias estadísticamente significativas entre las escalas objetiva y subjetiva en inclusión social en ninguno de los dos grupos de edad.

A diferencia de sus compañeros más jóvenes, los mayores con DI que formaron parte de este estudio, discreparon con los profesionales que les prestaban apoyos en lo que a bienestar material se refiere, otorgando puntuaciones significativamente más bajas a los distintos indicadores que configuran esta dimensión (Tabla 3). Un hecho que pudiera dar cuenta de este menor bienestar material percibido en los más mayores es su menor presencia en actividades de carácter ocupacional o productivo (Tabla 1). 


\begin{tabular}{|c|c|c|c|c|c|c|}
\hline \multirow{2}{*}{ Dimensión calidad de vida } & \multicolumn{3}{|c|}{ Menores de 50 años $(\mathrm{N}=91)$} & \multicolumn{3}{|c|}{ Mayores de 50 años $(\mathrm{N}=83)$} \\
\hline & Mediana & $\mathbf{Z}$ & Sig. & Mediana & $\mathbf{z}$ & Sig. \\
\hline Escala objetiva AU & 11 & \multirow{2}{*}{$-3,3$} & \multirow{2}{*}{, 001} & 11 & \multirow{2}{*}{$-4,1$} & \multirow{2}{*}{,000 } \\
\hline Escala subjetiva AU & 10 & & & 9 & & \\
\hline Escala objetiva BL & 12 & \multirow{2}{*}{$-4,7$} & \multirow{2}{*}{,000 } & 12 & \multirow{2}{*}{$-4,2$} & \multirow{2}{*}{,000 } \\
\hline Escala subjetiva BL & 9 & & & 9 & & \\
\hline Escala objetiva BM & 9 & \multirow{2}{*}{,- 48} & \multirow{2}{*}{,629 } & 9 & \multirow{2}{*}{$-2,6$} & \multirow{2}{*}{,008 } \\
\hline Escala subjetiva BM & 9 & & & 8 & & \\
\hline Escala objetiva BEF & 12 & \multirow{2}{*}{$-4,8$} & \multirow{2}{*}{,000 } & 12 & \multirow{2}{*}{$-4,9$} & \multirow{2}{*}{,000 } \\
\hline Escala subjetiva BEF & 9 & & & 8 & & \\
\hline Escala objetiva índice CdV & 101 & \multirow{2}{*}{$-4,3$} & \multirow{2}{*}{,000 } & 104 & \multirow{2}{*}{$-4,8$} & \multirow{2}{*}{,000 } \\
\hline Escala subjetiva índice CdV & 95 & & & 90,5 & & \\
\hline
\end{tabular}

AU: autodeterminación; IS: inclusión social; BL: bienestar laboral; BM: bienestar material; BEF: bienestar emocional y físico; BF: bienestar familiar.

Fuente: elaboración propia.

Además de analizar las diferencias que pudieran existir intra-grupo entre las puntuaciones subjetivas y objetivas, se llevó a cabo un contraste de medias para muestras independientes ( $U$ de Mann-Whitney) con el objetivo de identificar si existían diferencias estadísticamente significativas en la CdV de las personas que residían en las viviendas de Atzegi en función de su edad. Los resultados obtenidos pusieron de manifiesto que, sin tener en cuenta la posible interacción de cualquier otra variable, no existieron diferencias significativas entre personas mayores y menores de 50 años en lo que a su CdV se refiere cuando esta es medida de manera objetiva (Tabla 4). Aunque las personas mayores de 50 años con DI puntuaron su CdV de manera más baja que sus compañeros más jóvenes en casi todas las dimensiones que integran este constructo (especialmente en bienestar familiar y material), tampoco se observaron diferencias estadísticamente significativas entre grupos cuando la CdV fue valorada de forma subjetiva (Tabla 5).

Tabla 4. Diferencias en calidad de vida, medida de manera objetiva, entre mayores y menores de 50 años

\begin{tabular}{|l|c|c|c|c|}
\hline \multirow{2}{*}{ Dimensiones de calidad de vida } & $\begin{array}{c}\text { Menores de 50 años } \\
(\mathbf{N}=\mathbf{9 1})\end{array}$ & $\begin{array}{c}\text { Mayores de 50 años } \\
\text { (N = 83) }\end{array}$ & \multicolumn{2}{|c|}{ Sig. } \\
\cline { 2 - 5 } & Rango promedio & Rango promedio & $\mathbf{Z}$ &, 62 \\
\hline Autodeterminación & 85,7 & 89,4 &,- 50 &, 97 \\
\hline Inclusión social & 87,6 & 87,4 &,- 04 &, 74 \\
\hline Bienestar laboral & 86,5 & 88,6 &,- 33 &, 14 \\
\hline Bienestar material & 82,7 & 92,7 & $-1,5$ &, 53 \\
\hline Bienestar emocional y físico & 89,5 & 85,3 &,- 63 &, 81 \\
\hline Índice de calidad de vida & 86,6 & 88,4 &,- 24 & \\
\hline
\end{tabular}

Fuente: elaboración propia. 


\begin{tabular}{l}
\hline Tabla 5. Diferencias en calidad de vida, medida de manera subjetiva, entre mayores y menores de 50 años \\
\begin{tabular}{|l|c|c|c|c|}
\hline \multirow{2}{*}{ Dimensiones de calidad de vida } & $\begin{array}{c}\text { Menores de 50 años } \\
\text { (N = 91) }\end{array}$ & $\begin{array}{c}\text { Mayores de 50 años } \\
\text { (N = 83) }\end{array}$ & \multicolumn{2}{|c|}{ Sig. } \\
\cline { 2 - 5 } & Rango promedio & Rango promedio & Z &, 49 \\
\hline Autodeterminación & 86,4 & 81,4 &,- 68 &, 93 \\
\hline Inclusión social & 83,7 & 84,3 &,- 08 &, 91 \\
\hline Bienestar laboral & 83,6 & 84,4 &,- 10 &, 30 \\
\hline Bienestar material & 87,7 & 80,1 & $-1,03$ &, 37 \\
\hline Bienestar emocional y físico & 87,3 & 80,6 &,- 90 &, 16 \\
\hline Bienestar familiar & 89,1 & 78,7 & $-1,4$ &, 33 \\
\hline Índice de calidad de vida & 87,6 & 80,3 &,- 98 & \\
\hline
\end{tabular}
\end{tabular}

Fuente: elaboración propia.

Siendo conocida la influencia que determinadas variables pudieran ejercer en la CdV de los individuos, y, especialmente, en determinados momentos de su vida (como puede ser el cese de la actividad laboral durante la vejez), se presenta a continuación el análisis de la CdV de personas con DI mayores y menores de 50 años que residen en las viviendas de Atzegi teniendo en consideración las siguientes variables sociodemográficas: sexo, entorno de vivienda, actividad realizada durante el día, modificación de la capacidad legal de obrar, porcentaje de discapacidad y presencia de otras discapacidades y tipo de entorno (rural o urbano).

Análisis de la calidad de vida de mayores y menores de 50 años en función de determinadas variables sociodemográficas

\section{a. Sexo}

No se observaron diferencias estadísticamente significativas en función del sexo en las personas menores de 50 años en lo que a su CdV se refiere, encontrándose los valores de significación del estadístico $U$ de Mann-Whitney entre 108 (autodeterminación medida de manera objetiva) y 918 (índice general de CdV medida de manera objetiva).

Entre la población mayor de 50 años que residía en las viviendas de Atzegi, solo se observaron puntuaciones superiores y estadísticamente significativas para las mujeres en la dimensión de bienestar material medida de manera objetiva $(z=-2,14, p<, 05)$.

\section{b. Tipo de vivienda}

En función del tipo de vivienda en que residen las personas con DI, se observaron diferencias, no tanto en función de la edad, sino dependiendo de quién realizó la valoración de CdV. Así, aquellos menores de 50 años que residían en viviendas con mayor intensidad de apoyos recibían por parte de los profesionales puntuaciones significativamente más bajas en dimensiones de CdV como autodeterminación $(H(2)=22,2$, $p<, 001)$, inclusión social $(H(2)=12,4, p<, 01)$ y en el índice general de Calidad de Vida $(H(2)=9,5, p<, 01)$ que sus compañeros en viviendas de apoyo medio o intermitente. No obstante, cuando la calidad de vida fue valorada por las propias personas con DI menores de 50 años, solo se apreciaron diferencias estadís- 
ticamente significativas en la dimensión de bienestar emocional y físico $(H(2)=6,4, p<, 05)$, considerando aquellos que residían en pisos con mayor intensidad de apoyos que su bienestar era mayor.

En personas mayores de 50 años los resultados fueron muy similares. Cuando los profesionales realizaron la valoración, se apreciaron puntuaciones significativamente inferiores en la dimensión de autodeterminación $(H(2)=17,1, p<, 001)$ y en CdV general $(H(2)=7,8 p<, 05)$ para aquellos residiendo en viviendas con más intensidad de apoyos. En las escalas subjetivas, si bien es cierto que aquellos que vivían en entornos con mayor intensidad de apoyos consideraron que su bienestar emocional y físico era mayor, no se observaron diferencias estadísticamente significativas en ninguna dimensión en función del tipo de vivienda.

\section{c. Tipo de actividad realizada durante el día}

Además del tipo de vivienda en el que residen las personas, el tipo de actividad que realizan durante el día también pudiera afectar a su CdV. Tal y como queda reflejado en la Tabla 6, cuando la CdV fue medida de manera objetiva, las personas menores de 50 años que acudían a centro de día obtuvieron puntuaciones significativamente inferiores en las dimensiones de autodeterminación, inclusión social y bienestar material que aquellos que realizaban algún tipo de actividad ocupacional o productiva. En otras palabras, los profesionales percibían que el hecho de realizar una actividad ocupacional o productiva ejercía un impacto positivo en el grado en que las personas participaban en su entorno, tomaban decisiones y disponían de bienes materiales. Sin embargo, cuando fueron las propias personas con DI menores de 50 años quienes valoraron su CdV, este impacto positivo solo se observó en la dimensión Inclusión Social, manifestando incluso aquellos que contaban con un empleo un menor bienestar laboral $(M d n=7)$ y emocional y físico ( $M d n$ = 8) que aquellos en centro de día (bienestar laboral $M d n=9,50$; bienestar emocional y físico $M d n=9$ ) y centro ocupacional (bienestar laboral $M d n=10$; bienestar emocional y físico $M d n=10,5$ ). 
Tabla 6. Calidad de Vida de personas de menores de $\mathbf{5 0}$ años en función del tipo de actividad de día. Escala subjetiva y objetiva (prueba de Kruskal Wallis)

\begin{tabular}{|c|c|c|c|c|c|c|c|c|c|}
\hline \multirow[b]{2}{*}{ Dimensiones } & \multirow[b]{2}{*}{ Actividad diurna } & \multicolumn{4}{|c|}{ Calidad de vida objetiva } & \multicolumn{4}{|c|}{ Calidad de vida subjetiva } \\
\hline & & $\mathbf{N}$ & $\begin{array}{c}\text { Rango } \\
\text { promedio }\end{array}$ & $\mathbf{H}$ & Sig. & $\mathbf{N}$ & $\begin{array}{c}\text { Rango } \\
\text { promedio }\end{array}$ & $\mathbf{H}$ & Sig. \\
\hline \multirow{3}{*}{ Autodeterminación } & Empleo & 32 & 60,02 & \multirow{3}{*}{33,8} & \multirow{3}{*}{,00 } & 31 & 47,18 & \multirow{3}{*}{1,4} & \multirow{3}{*}{,49 } \\
\hline & C. Ocupacional & 36 & 50,33 & & & 36 & 40,53 & & \\
\hline & Centro de día & 23 & 19,72 & & & 18 & 40,75 & & \\
\hline \multirow{3}{*}{ Inclusión social } & Empleo & 32 & 50,02 & \multirow{3}{*}{9,3} & \multirow{3}{*}{,01 } & 31 & 39,29 & \multirow{3}{*}{7,1} & \multirow{3}{*}{,03 } \\
\hline & C. Ocupacional & 36 & 50,74 & & & 36 & 50,90 & & \\
\hline & Centro de día & 23 & 33,00 & & & 18 & 33,58 & & \\
\hline \multirow{3}{*}{ Bienestar laboral } & Empleo & 32 & 51,00 & \multirow{3}{*}{2,5} & \multirow{3}{*}{,28 } & 31 & 33,74 & \multirow{3}{*}{8,1} & \multirow{3}{*}{,02 } \\
\hline & C. Ocupacional & 36 & 43,40 & & & 36 & 50,60 & & \\
\hline & Centro de día & 23 & 43,11 & & & 18 & 43,75 & & \\
\hline \multirow{3}{*}{ Bienestar material } & Empleo & 32 & 51,89 & \multirow{3}{*}{6,6} & \multirow{3}{*}{,03 } & 31 & 39,94 & \multirow{3}{*}{2,5} & \multirow{3}{*}{,28 } \\
\hline & C. Ocupacional & 36 & 47,26 & & & 36 & 47,92 & & \\
\hline & Centro de día & 23 & 35,83 & & & 18 & 38,44 & & \\
\hline \multirow{3}{*}{$\begin{array}{l}\text { Bienestar } \\
\text { emocional y físico }\end{array}$} & Empleo & 32 & 44,20 & \multirow{3}{*}{1,8} & \multirow{3}{*}{,41 } & 31 & 37,18 & \multirow{3}{*}{6,3} & \multirow{3}{*}{,04 } \\
\hline & C. Ocupacional & 36 & 44,06 & & & 36 & 50,74 & & \\
\hline & Centro de día & 23 & 51,54 & & & 18 & 37,56 & & \\
\hline \multirow{3}{*}{ Bienestar familiar } & & & & & & 31 & 36,37 & \multirow{3}{*}{3,7} & \multirow{3}{*}{,16 } \\
\hline & & & & & & 36 & 46,61 & & \\
\hline & & & & & & 18 & 47,19 & & \\
\hline \multirow{3}{*}{ Índice de CdV } & Empleo & 32 & 57,45 & & & 31 & 36,29 & & \\
\hline & C. Ocupacional & 36 & 46,94 & 16,2 &, 00 & 36 & 49,97 & 5,3 & ,07 \\
\hline & Centro de día & 23 & 28,59 & & & 18 & 40,61 & & \\
\hline
\end{tabular}

Fuente: elaboración propia.

En las personas mayores de 50 años, los resultados fueron muy similares a los encontrados en menores de 50 años de edad cuando la CdV fue medida de manera objetiva y se tuvo en cuenta el tipo de actividad diurna realizada. Tal y como se refleja en la Tabla 7, cuando fueron los profesionales quienes realizaron la evaluación de la CdV de las personas con DI en distintos entornos de actividad, otorgaron puntuaciones significativamente superiores a aquellos en entornos productivos en las dimensiones de autodeterminación e inclusión social. Si bien las valoraciones de los profesionales no reflejaron diferencias estadísticamente significativas en función del tipo de actividad desempeñada por los mayores de 50 en la dimensión bienestar material (como sí ocurría en aquellos más jóvenes), consideraron que disponer de un empleo a estas edades contribuye de manera estadísticamente significativa a la mejora del bienestar laboral de las personas (Tabla 7). 
Cuando las personas mayores de 50 realizaron la valoración de su CdV, aquellos que solo acudían a centro de día refirieron puntuaciones significativamente más bajas en autodeterminación $(M d n=8)$ que quienes sí contaban con algún tipo de actividad ocupacional o laboral $(M d n=10)$.

\begin{tabular}{|c|c|c|c|c|c|c|c|c|c|}
\hline & & \multicolumn{4}{|c|}{ Calidad de vida objetiva } & \multicolumn{4}{|c|}{ Calidad de vida subjetiva } \\
\hline & Actividad diurna & $\mathbf{N}$ & $\begin{array}{c}\text { Rango } \\
\text { promedio }\end{array}$ & $\mathbf{H}$ & Sig. & $\mathbf{N}$ & $\begin{array}{c}\text { Rango } \\
\text { promedio }\end{array}$ & $\mathbf{H}$ & Sig. \\
\hline \multirow{3}{*}{ Autodeterminación } & Empleo & 24 & 62,77 & \multirow{3}{*}{26,2} & \multirow{3}{*}{, 00} & 24 & 51,54 & \multirow{3}{*}{6,5} & \multirow{3}{*}{,03 } \\
\hline & C. Ocupacional & 20 & 36,23 & & & 19 & 35,11 & & \\
\hline & Centro de día & 39 & 32,18 & & & 39 & 38,44 & & \\
\hline \multirow{3}{*}{ Inclusión social } & Empleo & 24 & 54,98 & \multirow{3}{*}{13,1} & \multirow{3}{*}{, 00} & 24 & 38,73 & \multirow{3}{*}{2,8} & \multirow{3}{*}{,24 } \\
\hline & C. Ocupacional & 20 & 33,10 & & & 19 & 49,45 & & \\
\hline & Centro de día & 39 & 38,58 & & & 39 & 39,33 & & \\
\hline \multirow{3}{*}{ Bienestar laboral } & Empleo & 24 & 51,83 & \multirow{3}{*}{8,5} & \multirow{3}{*}{, 01} & 24 & 39,06 & \multirow{3}{*}{,94 } & \multirow{3}{*}{,62 } \\
\hline & C. Ocupacional & 20 & 37,20 & & & 19 & 39,16 & & \\
\hline & Centro de día & 39 & 38,41 & & & 39 & 34,14 & & \\
\hline \multirow{3}{*}{ Bienestar material } & Empleo & 24 & 39,56 & \multirow{3}{*}{,98 } & \multirow{3}{*}{, 61} & 24 & 39,25 & \multirow{3}{*}{1,7} & \multirow{3}{*}{,41 } \\
\hline & C. Ocupacional & 20 & 40,10 & & & 19 & 41,74 & & \\
\hline & Centro de día & 39 & 44,47 & & & 39 & 39,85 & & \\
\hline \multirow{3}{*}{$\begin{array}{l}\text { Bienestar emocional } \\
\text { y físico }\end{array}$} & Empleo & 24 & 46,65 & \multirow{3}{*}{5,6} & \multirow{3}{*}{, 06} & 24 & 38,48 & \multirow{3}{*}{1,5} & \multirow{3}{*}{, 47} \\
\hline & C. Ocupacional & 20 & 48,10 & & & 19 & 41,03 & & \\
\hline & Centro de día & 39 & 36,01 & & & 39 & 40,67 & & \\
\hline \multirow{3}{*}{ Bienestar familiar } & & & & & & 24 & 37,69 & \multirow{3}{*}{,89 } & \multirow{3}{*}{,64 } \\
\hline & & & & & & 19 & 42,79 & & \\
\hline & & & & & & 39 & 43,22 & & \\
\hline \multirow{3}{*}{ Índice de CdV } & Empleo & 24 & 61,50 & \multirow{3}{*}{22,4} & & 24 & 37,69 & & \\
\hline & C. Ocupacional & 20 & 35,58 & &, 00 & 19 & 45,24 & 1,1 &, 57 \\
\hline & Centro de día & 39 & 33,29 & & & 39 & 42,03 & & \\
\hline
\end{tabular}

Fuente: elaboración propia.

\section{d. Tutela}

El análisis de la influencia de la modificación en la capacidad legal de obrar en las personas menores de 50 años que residían en las viviendas de Atzegi arrojó que el hecho de estar tutelado (bien por un familiar, bien por una fundación tutelar) daba lugar a que los profesionales valoraran de manera significativamente más baja las dimensiones de autodeterminación $(H(2)=10,15, p<, 01)$, inclusión social $(H(2)=7,75, p<, 05)$ y CdV general $(H(2)=6,18, p<, 05)$. Tales diferencias se observaron especialmente en aquellos tutelados por su familia (autodeterminación $M d n=10$; inclusión social $M d n=9$; índice de CdV Mdn =99,5) frente a aquellos tutelados por la propia Fundación (autodeterminación Mdn = 11; inclusión social Mdn = 12; índice de CdV Mdn=102) o sin tutelar (autodeterminación Mdn = 12; inclusión social $M d n=12$; índice de CdV Mdn = 107). 
Por el contrario, cuando las propias personas con DI menores de 50 años valoraron su CdV, la modificación en la capacidad legal de obrar solo repercutió negativamente en su bienestar familiar. Sobre todo, se apreciaron puntuaciones significativamente más bajas en esta dimensión $(H(2)=8,78, p<, 05)$ en aquellas personas que se encontraban tuteladas por la Fundación Tutelar Atzegi $(M d n=8)$ frente a aquellas tuteladas por su familia $(M d n=9)$ o sin tutelar $(M d n=10)$.

En las personas mayores de 50 años, se observó una tendencia similar. Se registraron así diferencias estadísticamente significativas en la dimensión de autodeterminación $(H(2)=20,03, p<, 001)$ cuando fueron los profesionales quienes cumplimentaron la escala, valorando esta dimensión peor en aquellos tutelados por su familia $(M d n=10)$ que en el resto de los grupos $(M d n=12)$.

Al igual que los profesionales, los mayores de 50 años tutelados por sus familias valoraron su autodeterminación de manera significativamente más baja $(H(2)=8,83, p<, 05)$ y, si bien los resultados en lo que a bienestar familiar se refiere no fueron estadísticamente significativos $(p=, 07)$, a diferencia de lo que ocurría en aquellos menores de 50 años, las personas tuteladas por la Fundación Tutelar Atzegi $(M d n=7)$ o por algún familiar $(M d n=8)$ refirieron puntuaciones más bajas en esta dimensión que aquellos sin tutelar $(M d n=9)$.

\section{e. Porcentaje de Discapacidad y presencia de otras discapacidades}

Solo cuando la CdV se valoró de manera objetiva en las personas menores de 50 años, el porcentaje de discapacidad ejerció cierta influencia en la CdV, concretamente en la dimensión de autodeterminación $(H(3)=$ $9,65, p<, 05)$, obteniendo aquellos con un porcentaje de discapacidad más alto (75\% o más) puntuaciones significativamente inferiores.

Una tendencia muy similar se observó en aquellos mayores de 50 años. Así, el porcentaje de discapacidad influyó en las puntuaciones de CdV de las personas solo cuando fueron los profesionales quienes cumplimentaron las escalas, presentando aquellos con un porcentaje de discapacidad superior (75\% o más) puntuaciones significativamente más bajas en la dimensión de autodeterminación $(H(3)=28,2, p<, 001)$ y en CdV general $(H(3)=18,68, p<, 001)$.

Cuando fueron las propias personas quienes valoraron su CdV, no se apreciaron diferencias estadísticamente significativas en función de esta variable en ninguno de los dos grupos de edad. Resulta preciso señalar, no obstante, que aquellas personas mayores de 50 años que, independientemente del porcentaje de discapacidad reconocido, presentaban otro tipo de discapacidad (física o enfermedad mental), referían puntuaciones significativamente más bajas en su bienestar laboral $(z=-2,91, p<, 01)$.

\section{f. Tipo de entorno (rural o urbano)}

No se observaron diferencias estadísticamente significativas en CdV en las personas menores de 50 años en función del entorno en que residían. No obstante, al analizar la CdV de las personas mayores de 50 años, los entornos más pequeños se relacionaron con puntuaciones significativamente más altas en inclusión social, medida tanto de manera objetiva $(z=-2,50, p<, 05)$ como subjetiva $(z=-2,36, p<, 05)$. 


\section{Conclusiones y discusión}

Como se ha expuesto en la introducción, es necesario reparar en las necesidades de las personas con DI que envejecen para poder prestar los apoyos necesarios en un amplio abanico de áreas vitales (Madrigal et al., 2007). Por este motivo, el presente trabajo ha analizado la CdV de las personas con DI menores y mayores de 50 años usuarias del servicio de viviendas de Atzegi, con ánimo de detectar necesidades que pudieran surgir en edades más avanzadas y que debieran ser tenidas en cuenta en el diseño de planes más centrados en la persona.

Respecto a este primer objetivo general es importante destacar que no se apreciaron diferencias estadísticamente significativas entre los mayores y menores de 50 años en lo que a su CdV se refiere cuando el único factor explicativo a tener en cuenta en posibles discrepancias fue la edad. Así, a pesar de que los más mayores percibían su CdV algo más baja que la de sus compañeros más jóvenes, no se observaron diferencias estadísticamente significativas entre grupos, ni cuando fueron las propias personas quienes valoraron su CdV (escala subjetiva), ni cuando lo hicieron los profesionales (escala objetiva). No obstante, los análisis realizados han puesto de manifiesto que otros factores más allá de la edad de la persona con DI parecen condicionar los resultados personales. Por sus implicaciones en la prestación de apoyos, nos centraremos en tres de ellos: el tipo de vivienda, el tipo de actividad realizada y la modificación en la capacidad legal de obrar.

Con respecto a la primera variable, el entorno en el que residen las personas con DI parece ejercer cierta influencia en cómo su CdV es percibida por ellas mismas y por los profesionales que les prestan apoyos. Si bien no se observaron diferencias notables entre grupos de edad en función del tipo de vivienda, los profesionales sí parecen percibir que, independientemente de la edad, el hecho de precisar de menos apoyo afecta de manera positiva a la autodeterminación e inclusión social de las personas con DI, repercutiendo positivamente en su CdV general. Dada la inexistencia de diferencias estadísticamente significativas entre grupos en función de la edad en los distintos entornos de vivienda, los profesionales pudieran estar considerando que es la variable 'intensidad de los apoyos requeridos' lo que pudiera condicionar resultados positivos en CdV (concretamente en las dimensiones de autodeterminación e inclusión social). Estudios previos como el de Bigby et al. (2009) ponen de manifiesto cómo la intensidad de los apoyos que se requieren condiciona la visión que los profesionales tienen sobre posibilidades de autodeterminación e inclusión social.

Cuando fueron las propias personas con DI quienes valoraron su CdV, tales diferencias entre grupos en función del tipo de vivienda no se observaron. No obstante, tanto mayores como menores de 50 años que residían en viviendas con atención más continuada, refirieron un mayor bienestar emocional y físico. Si tenemos en cuenta que el bienestar físico es una de las dimensiones más priorizadas por las personas con DI que presentan mayor necesidad de apoyos (Kreuger et al., 2008), el contar con un entorno que intensifique las interacciones de los profesionales con la persona con DI, podría estar contribuyendo a este resultado positivo (Maes et al., 2007). En este sentido, estudios recientes sobre apoyo activo ponen de manifiesto que el tipo de apoyo que se presta constituye una de las variables más relevantes a la hora de conseguir un incremento en el bienestar de la persona (Beadle-Brown et al., 2015, citado por Verdugo y Navas, 2017). Analizar si las diferencias percibidas en CdV en función del tipo de vivienda se deben, no tanto a la edad, sino a diferentes formas de prestar apoyo, debe constituir un reto futuro para esta y otras organizaciones del 
tercer sector de acción social de nuestro país. Evitaríamos así el traslado de personas que alcanzan la edad de 65 años y que quieren seguir residiendo en su entorno habitual a residencias de tercera edad, situación que ya está teniendo lugar en comunidades autónomas de nuestro país al considerarla más apropiada para dar respuesta a las necesidades de aquellos que envejecen y que se fundamenta más en una decisión de tipo administrativo que en una decisión centrada en la persona.

Además del tipo de vivienda, el tipo de actividad diurna fue otra de las variables que influyó en la CdV de las personas con DI. Fue llamativo observar cómo los menores de 50 años percibían un menor bienestar laboral, emocional y físico cuando contaban con un empleo que cuando realizan una actividad ocupacional o de día. La mayor exigencia de las demandas en entornos productivos, pudiera, como ya han señalado otros autores (Flores et al., 2010) explicar estos resultados. Sin embargo, las valoraciones realizadas por los mayores de 50 años que aún trabajaban no arrojaron estos resultados. Es más, las personas mayores de 50 años, refirieron mayores niveles de autodeterminación si realizaban una actividad productiva. Quizá el hecho de desempeñar una actividad laboral sea especialmente valorado por aquellos que se encuentran más cerca del cese de su actividad, quienes desean continuar participando en actividades que sigan favoreciendo su desarrollo personal (Berzosa, 2013; Bigby, 2004; Elorriaga et al., 2012). Organizaciones de nuestro entorno como Lantegi Batuak (Elorriaga et al., 2012) advierten sobre la necesidad de mantener a las personas con DI que envejecen con el mayor nivel de actividad posible para prevenir los efectos de deterioro, desarrollando para ello un sistema de organización de apoyos a la actividad laboral que debiera combinarse con un detallado plan de transición que priorice la participación de la persona en actividades de su interés durante y tras el cese de la actividad laboral (Stancliffe et al., 2015). Además, deberá prestarse especial atención a los apoyos adicionales que pudieran requerir aquellos que presentan otro tipo de discapacidad asociada, quienes en el presente trabajo refieren puntuaciones más bajas en la dimensión de bienestar laboral.

La CdV de las personas con DI también parece verse influida por el hecho de estar o no tutelado, ya que las personas tanto menores como mayores de 50 años que se encuentran tuteladas por sus familias o por la Fundación Tutelar Atzegi presentaron peores puntuaciones en CdV, concretamente en las dimensiones de autodeterminación y bienestar familiar. Tal y como afirman Madrigal et al. (2007), suele ser frecuente encontrar a familias que evitan que las personas con DI vivan ciertas experiencias negativas como pueden ser situaciones de enfermedad o la muerte de seres queridos, promoviendo, aunque sea de manera no intencionada, la infantilización de este colectivo y la menor participación en la toma de decisiones. Desde el servicio de viviendas se ha de que prestar atención a este aspecto y hacer partícipe a la persona con DI de todas las decisiones que se tomen, tenga o no su capacidad de obrar modificada, estableciendo a la vez estrechos lazos de colaboración con las familias y tutores que faciliten un proceso de apoyo a la toma de decisiones por parte de la persona con DI. Asimismo, estos resultados debieran llevarnos a intentar fomentar el sentimiento de familia dentro de la organización. Una futura línea de trabajo en este sentido consiste en fortalecer la iniciativa de delegados tutelares para las personas que se encuentran tuteladas por la Fundación. Dicha iniciativa trata de buscar figuras de apoyo y de referencia que queden con la persona con DI y realicen diversas actividades que sean enriquecedoras para la misma. El hecho de tener a una persona de referencia puede suplir de alguna manera ese sentimiento de vacío familiar. Las experiencias llevadas a cabo hasta el momento con esta figura dentro de la organización han resultado enriquecedoras para las personas.

El estudio realizado supone un punto de partida para comenzar las mencionadas líneas futuras de trabajo dentro de la organización y prestar algo más de atención a aquellas variables que, como hemos visto, pu- 
dieran ejercer un impacto negativo en la CdV de la persona. Para ello será necesario, no obstante, solventar algunas de las limitaciones de las que adolece el presente estudio.

En primer lugar, son diversas las variables que pueden afectar a la CdV de las personas con DI que no aparecen recogidas en el presente trabajo. Hemos incluido aquí variables fácilmente observables y susceptibles de medida, como pudieran ser el tipo de entorno, el tipo de actividad laboral o la existencia o no de una modificación en la capacidad legal de obrar, pero la CdV se ve influida por otros muchos aspectos de carácter subjetivo que no se recogen en este estudio, como la red de apoyo social (Nolan et al., 2001), las oportunidades para el desarrollo personal (Judge et al., 2010), o la satisfacción personal y autoconcepto (Schalock y Verdugo, 2002), entre otros muchos factores.

Además, en futuros trabajos, se ha de procurar recoger la variable edad de manera continua. Al haber recogido esta variable en rangos de edad predeterminados, no se han podido realizar análisis estadísticos que requerían contar con la edad cronológica de la persona. Por otro lado, el hecho de no disponer apenas de datos sobre población mayor de 65 años, obliga a recomendar cautela a la hora de considerar estos datos como representativos de las personas con DI más envejecidas.

El pequeño tamaño de muestra en las categorías de algunas variables, impide en el momento actual analizar la interacción que pudiera existir entre las mismas en distintos grupos de edad, así como su poder predictivo en la CdV de los usuarios, siendo necesario, de cara al futuro, extender este estudio a otras organizaciones de nuestro entorno, con el objetivo último de ejercer un mayor control en este sentido y garantizar una mejor representatividad de todas aquellas variables cuyo efecto en la CdV se desee analizar.

En cuanto a otras limitaciones del trabajo, quisiéramos hacer referencia a la escala utilizada para este estudio, la Escala Integral de Calidad de Vida (Verdugo et al., 2009). A pesar de ser una escala de gran utilidad, es importante destacar que la Escala INICO-FEAPS (Verdugo et al., 2013) es un instrumento cuyo origen se encuentra en la Escala Integral, mejorando ampliamente su contenido, propiedades psicométricas y utilidad. Pero tal y como se ha comentado anteriormente, Atzegi lleva años utilizando la Escala Integral de Calidad de Vida y, a pesar de que una mejora planteada para este último año ha sido introducir la Escala INICO-FEAPS en los planes personales de apoyo, resultaba necesario analizar los datos recogidos durante un periodo de dos años con la Escala Integral, cuyas propiedades psicométricas también nos permiten garantizar la fiabilidad y validez de los datos presentados (Verdugo et al., 2009). Por otra parte, el empleo de otros instrumentos como la Escala San Martín (Verdugo et al., 2014) pudiera haber solventado las dificultades encontradas en la evaluación de calidad de vida de aquellas personas con mayores dificultades de expresión y comprensión.

A pesar de las limitaciones señaladas, el presente trabajo permitirá poner en marcha estrategias de mejora en la organización orientadas a mejorar la CdV de sus usuarios, ofreciéndonos pistas iniciales sobre aquellos factores que pudieran contribuir a vivir un proceso de envejecimiento más activo y satisfactorio. 


\section{Referencias bibliográficas}

Aguado, A. y Alcedo, A. (2004): "Necesidades percibidas en el proceso de envejecimiento de las personas con discapacidad". Psicothema, 16 (2): 261-269.

Baroja, H. et al. (2014): Envejecimiento y discapacidad intelectual. Bilbao: FEVAS.

Beadle-Brown, J. et al. (2015): "Observing practice leadership in intellectual and developmental disability services”. Journal of Intellectual Disability Research, 59 (12): 1081-1093.

Berjano, E. y García, E (2010): Discapacidad intelectual y envejecimiento: un problema social del siglo XXI. Madrid: FEAPS.

Berzosa, G. (2013): Las personas con síndrome de Down y sus familias ante el proceso de envejecimiento. Madrid: Real Patronato sobre Discapacidad.

Bigby, C. (2008): "Beset by obstacles: a review of australian policy development to support ageing in place for people with intellectual disability”. Journal of Intellectual and Developmental Disability, 33 (1): 76-86.

Bigby, C. (2004): Ageing with a lifelong disability: a guide to practice, program and policy issues for human services professionals. Londres: Jessica Kingsley Publishers.

Bigby, C. et al. (2012): Making life good in the community. Measures of resident outcomes and staff perceptions of the move from an institution (en línea). <http://arrow.latrobe.edu.au:8080/vital/access/manager/Repository/ latrobe:34103>, acceso 7 de diciembre de 2018.

Bigby, C. et al. (2009): “'It's pretty hard with our ones, they can't talk, the more able bodied can participate': Staff attitudes about the applicability of disability policies to people with severe and profound intellectual disabilities". Journal of Intellectual Disability Research, 53 (4): 363-376.

Bigby, C. y Beadle-Brown, J. (2018): "Improving quality of life outcomes in supported accommodation for people with intellectual disability: What makes a difference?". Journal of Applied Research in Intellectual Disabilities, 31 (2): 182-200.

Brickell, C. y Munir, K. (2008): "Grief and its complications in individuals with intellectual disability”. Harvard Review of Psychiatry, 16 (1): 1-12.

Carratalá, A. (2017): Planificación centrada en la persona: planificando por adelantado el futuro deseado. Madrid: Plena Inclusión.

Cristóbal, L. et al. (2017): "Duelo en discapacidad intelectual: los avances de una década". Revista Española de Discapacidad, 5 (2): 53-72.

Elorriaga E. et al. (2012): "Envejecimiento y deterioro de las personas con discapacidad intelectual de Lantegi Batuak en el ámbito ocupacional y del empleo en Bizkaia”. Zerbitzuan, 51: 119-135.

Flores, N. et al. (2010): "Análisis de la calidad de vida laboral en trabajadores con discapacidad." Zerbitzuan, 47: 95-107.

Grammenos, S. (2013): European comparative data on Europe 2010 \& people with disabilities. Final Report. Academic Network of European Disability Experts (ANED) (en línea). <https://digitalcommons.ilr.cornell.edu/cgi/ viewcontent.cgi?article=1569\&context=gladnetcollect $>$, acceso 7 de diciembre de 2018 . 
Grupo de Trabajo de Envejecimiento Activo del CERMI Estatal (2012): El envejecimiento de las personas con discapacidad. Madrid: CINCA.

Haveman, M. et al. (2010): "Major health risks in aging persons with intellectual disabilities: An overview of recent studies". Journal of Policy and Practice in Intellectual Disabilities, 7 (1): 59-69.

Heller, T. et al. (2011): "Self-determination across the life span: Issues and gaps. Exceptionality, 19 (1): 31-45.

Heller, T. y Sorensen, A. (2013): "Promoting healthy aging in adults with developmental disabilities". Developmental Disabilities Research Reviews, 18: 22-30.

Higgins, L. y Mansell, J. (2009): "Quality of life in group homes and older persons' homes". British Journal of Learning Disabilities, 37 (3): 207-212.

Jokinen, N. et al. (2013): "Guidelines for structuring community care and supports for people with intellectual disabilities affected by dementia". Journal of Policy and Practice in Intellectual Disabilities, 10 (1): 1-24.

Judge, J. et al. (2010): "Activity, aging, and retirement: The views of a group of Scottish people with intellectual disabilities". Journal of Policy and Practice in Intellectual Disabilities, 7 (4): 295-301.

Kingsbury, L. A. (2009): People planning ahead. Washington: American Association on Intellectual and Developmental Disabilities.

Kirkendall, A. et al. (2017): "Intellectual disabilities and decision making at end of life: A literature review”. Journal of Applied Research in Intellectual Disabilities, 30 (6): 982-994.

Kreuger, L. et al. (2008): "Needs of persons with severe intellectual disabilities: a Q-methodological study of clients with severe behavioural disorders and severe intellectual disabilities". Journal of Applied Research in Intellectual Disabilities, 21 (5): 466-476.

Madrigal, A. et al. (2007): "Envejecimiento de las Personas con Discapacidad Intelectual”. Boletín sobre Envejecimiento, 26: 1-39.

Maes, B. et al. (2007): Quality-enhancing interventions for people with profound intellectual and multiple disabilities: A review of the empirical research literature. Journal of Intellectual and Developmental Disability, 32 (3): 163-178.

Mansell, J. (2010): Raising our sights: services for adults with profound intellectual and multiple disabilities (en línea).

Mansell, J. (2006) "Deinstitutionalisation and community living: progress, problems and priorities". Journal of Intellectual and Developmental Disability, 31: 65-76.

<https://www.mencap.org.uk/sites/default/files/2016-06/Raising_our_Sights_report.pdf>, acceso 7 de diciembre de 2018.

Navas, P. et al. (2014): Envejecimiento activo y discapacidad intelectual. Madrid: Ministerio de Educación, Cultura y Deporte.

Nolan, M. et al. (2001): Working with older people and their families. Philadelphia: McGraw-Hill International.

Novell, R. et al. (2008): Informe Séneca. Envejecimiento y discapacidad intelectual en Cataluña. Barcelona: FEAPS.

Pereyra, C. (2007): "Propuestas de FEAPS para el envejecimiento saludable de las personas con discapacidad intelectual”. Boletín sobre el Envejecimiento, 26:17-36. 
Presson, A. P. et al. (2013): "Current estimate of Down syndrome population prevalence in the United States". The Journal of Pediatrics, 163 (4): 1163-1168.

Reppermund, S. y Trollor, J. N. (2016): "Successful ageing for people with an intellectual disability". Current Opinion in Psychiatry, 29 (2): 149-154.

Schalock, R. L. et al. (2006): Calidad de Vida para personas con discapacidad intelectual y otras discapacidades del desarrollo; aplicaciones para personas, organizaciones, comunidades y sistemas. Madrid: Confederación Española de Organizaciones en favor de las Personas con Discapacidad Intelectual.

Shalock, R. L. y Verdugo, M.A. (2010): "Últimos avances en el enfoque y concepción de las personas con discapacidad intelectual”. Siglo Cero, 41 (4): 7-21.

Schalock, R. L. y Verdugo, M.A. (2002): Quality of life for human service practitioners. Washington, DC: American Association on Mental Retardation.

Stancliffe, R. J. et al. (2015): "Transition to retirement and participation in mainstream community groups using active mentoring: A feasibility and outcomes evaluation with a matched comparison group". Journal of Intellectual Disability Research, 59 (8): 703-718.

Stancliffe, R. J. et al. (2013): Transition to retirement: A guide to inclusive practice. Sydney University Press.

Verdugo, M. A. et al. (2014): "Measuring quality of life in people with intellectual and multiple disabilities: Validation of the San Martín scale”. Research in Developmental Disabilities, 35 (1): 75-86.

Verdugo, M. A. et al. (2013): Escala Inico-Feaps: evaluación Integral de la calidad de vida de personas con discapacidad intelectual o del desarrollo. Salamanca: INICO.

Verdugo, M. A., et al. (2009): Escala integral: evaluación objetiva y subjetiva de la calidad de vida de personas con discapacidad intelectual. Madrid: CEPE.

Verdugo, M.A. y Navas, P. (2017): Todos somos todos: derechos y calidad de vida de las personas con discapacidad intelectual y mayores necesidades de apoyo. Madrid: Real Patronato sobre Discapacidad.

Wark, S. et al. (2016): "The main signs of ageing in people with intellectual disability". Australian Journal of Rural Health, 24 (6): 357-362.

Whitley, E. y Ball, J. (2002): "Statistics review 6: Nonparametric methods". Critical Care, 6 (6): 509-513.

Wiese, M. et al. (2014): "What is talked about? Community living staff experiences of talking with older people with intellectual disability about dying and death". Journal of Intellectual Disability Research, 58 (7): 679-690. 